\title{
Spectral inequalities for operators on H-type groups
}

\author{
James Inglis ${ }^{1}$
}

\begin{abstract}
In this paper the spectra of certain Schrödinger-type operators defined in the sub-elliptic setting of H-type groups are investigated. Two approaches are taken: the first one makes use of a convenient unitary transformation on the Heisenberg group, while the second proceeds via functional inequalities. The paper concludes by highlighting a major difference between the spectrum of the considered operators depending on which natural sub-Riemannian metric is chosen.
\end{abstract}

Mathematics Subject Classification (2010). Primary 58J50; Secondary 22E30.

Keywords. H-type group, super-Poincaré inequality, spectral gap inequality, discrete spectrum, logarithmic Sobolev inequality.

\section{Introduction}

In the classical setting of $\mathbb{R}^{n}$ an extensive study has been made of the spectral properties of so-called Schrödinger operators, that is operators of the form

$$
\mathscr{L}=-\Delta+V
$$

where $\Delta$ is the standard Laplacian on $\mathbb{R}^{n}$ and $V$ is some potential. In particular the location of the continuous and discrete parts of the spectra of such operators (if indeed they exist) is of great interest. A classical reference detailing this study is the book of M. Reed and B. Simon [21].

In this paper we pursue the same questions, but instead work in the sub-Riemannian setting of H-type groups. We consider a direct analogue of the classical operator (1.1) in such a setting, where we replace the full Laplacian with the more natural subLaplacian. Given an H-type group $\mathbb{G}$, we will thus be interested in the sub-elliptic operators

$$
\mathscr{L}=-\Delta_{\mathbb{G}}+\nabla_{\mathbb{G}} U \cdot \nabla_{\mathbb{G}}
$$

\footnotetext{
${ }^{1}$ Supported by EPSRC EP/D05379X/1. Work was carried out while a member of Imperial College London, UK.
} 
where $\Delta_{\mathbb{G}}$ and $\nabla_{\mathbb{G}}$ are the sub-Laplacian and sub-gradient respectively. When considered as an operator acting on $L^{2}\left(\mu_{U}\right)$ with $\mu_{U} \equiv Z^{-1} e^{-U} d x$, such operators are positive and self-adjoint. Our aim will be principally to find conditions on the potential $U$ that ensure the operator (1.2) has a purely discrete spectrum, focusing on the natural case when $U$ is given by a power of one of the intrinsic distance functions defined on the group.

Our first attempt to answer some of these questions involves the use of an appealing unitary transformation on the Heisenberg group, but it quickly becomes apparent that such an approach is limited when it comes to general H-type groups and our preferred choices for the potential $U$. The majority of this paper is therefore devoted to taking a functional inequality approach, similar to that taken in [7], [25] and [28], in order to overcome these limitations.

Functional inequalities on $\mathrm{H}$-type groups have attracted considerable attention recently (see for example [1], [8], [9], [10], [17], and [18]), since despite being relatively simple cases of sub-Riemannian settings, the structure that they possess renders traditional methods inapplicable. Indeed, the main difficulty (as described in [1]) stems from the fact that the Ricci tensor of the sub-Laplacian on an H-type group is $-\infty$, and hence the methods of Bakry and Emery [2] to prove many useful inequalities can not be used.

Another major motivation for this paper is the work of Hebisch and Zegarfiński [14], in which an effective technology was introduced to prove inequalities such as the logarithmic Sobolev inequality for measures on H-type groups of the form

$$
\mu(d x)=\frac{e^{-\alpha d^{p}(x)}}{Z},
$$

where $\alpha>0, p>1$ and $d$ is Carnot-Carathéodory distance on the group. The work we present here can thus be thought of as an extension of their study to alternative inequalities that carry spectral information.

Similar questions to those addressed here have recently been investigated in [27], where general conditions for empty essential spectrum for hypoelliptic generators are put forward. However, these conditions are not easily applicable to the cases of special interest to us, i.e. when the potential $U$ in (1.2) is defined in terms of a natural distance function on the group. This is because of the singularities possessed by such distance functions, and hence our approach is necessarily different.

The layout of this paper is as follows. After presenting the necessary definitions and notation, we start by proving a generalisation of a classical result in the setting of the Heisenberg group, which gives some conditions on the growth and smoothness of the potential $U$ that ensure that the operator has empty essential spectrum. Since these conditions do not cover cases of particular interest to us and the methods are not easily generalised to all H-type groups, we then take a different approach, and investigate functional inequalities for the associated measures that contain spectral information. This section is split into two subsections: the first concentrates on the situation when the potential $U$ is defined in terms of the Carnot-Carathéodory distance, while 
the second tries to overcome some of the extra difficulties encountered when the Carnot-Carathéodory distance is replaced by the Kaplan distance. These difficulties are perhaps rather surprising, and it is interesting that they result in operators with differently structured spectra.

Acknowledgements. The work of this paper came about as a result of some discussions with Prof. L. Saloff-Coste, Prof. A. Laptev, and Prof. W. Hebsich to whom the author is very grateful.

\section{Definitions and notation}

In this section we briefly give the basic definitions and results that will be used throughout the rest of the paper. We refer the reader to [6] for a comprehensive review of this material.

Definition 2.1 (H-type group). Let $\mathfrak{g}$ be a finite-dimensional real Lie algebra equipped with Lie bracket $[\cdot, \cdot]: \mathfrak{g} \times \mathfrak{g} \rightarrow \mathfrak{g}$. Let $\mathfrak{z}$ denote the centre of $\mathfrak{g}$, that is $\mathfrak{z}=$ $\{X \in \mathfrak{g}:[X, Y]=0 \forall Y \in \mathfrak{g}\}$.

The Lie algebra $\mathfrak{g}$ is said to be of $H$-type if it can be endowed with an inner product $\langle\cdot, \cdot\rangle$ such that $\left[\mathfrak{z}^{\perp}, \mathfrak{z}^{\perp}\right]=\mathfrak{z}$, and moreover, for every fixed $Z \in \mathfrak{z}$, the map $J_{Z}: \mathfrak{z}^{\perp} \rightarrow \mathfrak{z}$ defined by

$$
\left\langle J_{Z}(X), Y\right\rangle=\langle Z,[X, Y]\rangle \quad \forall Y \in \mathfrak{z}^{\perp}
$$

is an orthogonal map.

An H-type group is a connected and simply connected Lie group $\mathbb{G}$ whose Lie algebra is of H-type.

In addition to this abstract definition, we have the following characterisation result (Theorem 18.2.1 of [6]):

Theorem 2.2. $\mathbb{G}$ is an H-type group if and only if $\mathbb{G}$ is (isomorphic to) $\mathbb{R}^{n+m}$ with the group law

$$
(w, z) \circ(\omega, \zeta)=\left(\begin{array}{c}
w_{i}+\omega_{i}, \quad i=1, \ldots, n \\
z_{j}+\zeta_{j}+\frac{1}{2}\left\langle U^{(j)} w, \omega\right\rangle, \quad j=1, \ldots, m
\end{array}\right),
$$

for $w, \omega \in \mathbb{R}^{n}, z, \zeta \in \mathbb{R}^{m}$ and where the matrices $U^{(1)}, \ldots, U^{(m)}$ have the following properties:

(1) $U^{(j)}$ is an $n \times n$ skew-symmetric and orthogonal matrix for every $j \in\{1, \ldots, m\}$;

(2) $U^{(k)} U^{(j)}+U^{(j)} U^{(k)}=0$ for every $k, j \in\{1, \ldots, m\}$ with $k \neq j$. 
Thus, without any loss of generality, we will henceforth assume that any H-type group $\mathbb{G}$ is of this form. For an H-type group $\mathbb{G}$ and $x \in \mathbb{G}$, we will therefore use the notation

$$
x=(w, z)=\left(w_{1}, \ldots, w_{n}, z_{1}, \ldots, z_{m}\right),
$$

for $w \in \mathbb{R}^{n}$ and $z \in \mathbb{R}^{m}$. It is clear that the point $(0,0)$ is the identity in $\mathbb{G}$ and the inverse operation is $(w, z)^{-1}=(-w,-z)$.

There is a natural family of dilation operators on an $\mathrm{H}$-type group $\mathbb{G}$ :

Definition 2.3. The family of operators $\delta_{\lambda}: \mathbb{G} \rightarrow \mathbb{G}$ for $\lambda>0$ defined by

$$
\delta_{\lambda}(w, z)=\left(\lambda w, \lambda^{2} z\right)
$$

for $(w, z) \in \mathbb{G}$ is called the family of dilations on $\mathbb{G}$. The map $\delta_{\lambda}$ is a group homomorphism, in the sense that $\delta_{\lambda}(x \circ y)=\delta_{\lambda}(x) \circ \delta_{\lambda}(y), \forall x, y \in \mathbb{G}$.

We can identify $\mathfrak{g}$ with the space spanned by the left-invariant vector fields $\left\{X_{1}, \ldots, X_{n}, Z_{1}, \ldots, Z_{m}\right\}$ on $\mathbb{G}$, where

$$
X_{i}=\frac{\partial}{\partial w_{i}}+\frac{1}{2} \sum_{k=1}^{m} \sum_{l=1}^{n} U_{i l}^{(k)} w_{l} \frac{\partial}{\partial z_{k}}, \quad Z_{j}=\frac{\partial}{\partial z_{j}},
$$

for $i \in\{1, \ldots, n\}, j \in\{1, \ldots, m\}$. One can calculate that

$$
\left[X_{i}, X_{j}\right]=\sum_{k=1}^{m} U_{j i}^{(k)} Z_{k}
$$

from which it follows that $\operatorname{span}\left\{X_{i},\left[X_{j}, X_{k}\right]: i, j, k \in\{1, \ldots n\}\right\}=\mathfrak{g}$. This is equivalent to saying that the H-type group $\mathbb{G}$ is a Carnot group of step 2.

Definition 2.4. The second order differential operator $\Delta_{\mathbb{G}}=\sum_{j=1}^{n} X_{i}^{2}$ is the canonical sub-Laplacian on $\mathbb{G}$. The vector-valued operator $\nabla_{\mathbb{G}}=\left(X_{1}, \ldots, X_{n}\right)$ is the canonical sub-gradient on $\mathbb{G}$.

We will treat a general H-type group $\mathbb{G}$ as a metric space, and we can do this in two natural ways. Despite the fact that both metrics are equivalent (in fact all homogeneous metrics on an H-type group are equivalent — see Proposition 5.1.4 of [6]), it turns out that the choice of metric will make a big difference to the spectrum of the associated operator.

The first, and perhaps most common way to define a metric on an H-type group $\mathbb{G}$ is to exploit the structure of the group:

Definition 2.5 (Carnot-Carathéodory distance). Let $\gamma:[0,1] \rightarrow \mathbb{G}$ be an absolutely continuous path. We say that $\gamma$ is horizontal if there exist measurable functions 
$a_{1}, \ldots, a_{n}:[0,1] \rightarrow \mathbb{R}$ such that

$$
\dot{\gamma}(t)=\sum_{i=1}^{n} a_{i}(t) X_{i}(\gamma(t))
$$

for almost all $t \in[0,1]$ i.e. $\dot{\gamma}(t) \in \operatorname{span}\left\{X_{1}(\gamma(t)), \ldots, X_{n}(\gamma(t))\right\}$ almost everywhere. For such a horizontal curve $\gamma$, we define the length of $\gamma$ to be

$$
|\gamma|:=\int_{0}^{1}\left(\sum_{i=1}^{n} a_{i}^{2}(t)\right)^{\frac{1}{2}} d t .
$$

We then define the Carnot-Carathéodory distance $d(x, y)$ between two points $x, y \in$ $\mathbb{G}$ to be

$d(x, y):=\inf \{|\gamma|$ such that $\gamma:[0,1] \rightarrow \mathbb{G}$ is horizontal and $\gamma(0)=x, \gamma(1)=y\}$.

We will write $d(x):=d(x, 0)$.

It should be noted that the Carnot-Carathéodory distance is well-defined by Chow's Theorem (see for example [4] or [24]).

The second natural distance function that will be of interest to us arises from the fundamental solution of the sub-Laplacian $\Delta_{\mathbb{G}}$. This was discovered by A. Kaplan in [15] on general H-type groups, extending the work of G.B. Folland [11] on the Heisenberg group.

Definition 2.6 (Kaplan distance). Define the function $N: \mathbb{G} \rightarrow[0, \infty)$ by $N(x)=$ $\left(|w|^{4}+16|z|^{2}\right)^{1 / 4}$ for $x=(w, z) \in \mathbb{G}$. Then $N$ is a symmetric homogeneous norm, which we will call the Kaplan distance. Moreover, $F=N^{2-Q}$ is a fundamental solution of $\Delta_{\mathbb{G}}$, where $Q=2 m+n$ is the homogeneous dimension of the group, in the sense that $F$ is smooth out of the origin and

$$
\Delta_{\mathbb{G}} F(x)=0 \text { in } \mathbb{G} \backslash\{0\} .
$$

An important difference between the two distance functions is that $N$ is smooth everywhere outside the origin, whilst $d$ has points of non-smoothness on the centre of the group:

Proposition 2.7. (i) $d: \mathbb{G} \rightarrow[0, \infty)$ is smooth on the $\operatorname{set}\{x=(w, z) \in \mathbb{G}: w \neq 0\}$, and $\left|\nabla_{\mathbb{G}} d(x)\right|=1$, for all $x=(w, z) \in \mathbb{G}$ such that $w \neq 0$.

(ii) $N: \mathbb{G} \rightarrow[0, \infty)$ is smooth on $\mathbb{G} \backslash\{0\}$, and $\left|\nabla_{\mathbb{G}} N(x)\right|=\|x\| / N(x)$, for all $x=(w, z) \in \mathbb{G}$ such that $x \neq 0$, where $\|x\|:=|w|=\left(\sum_{i=1}^{n} w_{i}^{2}\right)^{\frac{1}{2}}$.

Proof. Part (i) is found in [19], whilst part (ii) can be calculated directly. 
Example 2.8 (The Heisenberg group). The main example of an H-type group to keep in mind is the general $n$-th Heisenberg group $\mathbb{H}^{n}$. In fact H-type groups were introduced as a generalisation of the Heisenberg group. $\mathbb{T}^{n}$ can be realised as $\mathbb{R}^{2 n+1}$ with the group operation

$$
(w, z) \circ(\omega, \zeta)=\left(w+\omega, z+\zeta-\frac{1}{2} \sum_{j=1}^{n}\left(w_{2 j-1} \omega_{2 j}-w_{2 j} \omega_{2 j-1}\right)\right)
$$

for $w=\left(w_{i}\right), \omega=\left(\omega_{i}\right) \in \mathbb{R}^{2 n}$, and $z, \zeta \in \mathbb{R}$. The left-invariant vector fields on $\mathbb{H}^{n}$ are given by

$$
X_{2 j-1}=\partial_{w_{2 j-1}}+\frac{1}{2} w_{2 j} \partial_{z}, \quad X_{2 j}=\partial_{w_{2 j}}-\frac{1}{2} w_{2 j-1} \partial_{z}, \quad X_{2 n+1}=\partial_{z},
$$

for $j=1, \ldots n$, and one can easily calculate that $\left[X_{2 j-1}, X_{2 j}\right]=-X_{2 n+1}$ and $\left[X_{2 j-1}, X_{2 n+1}\right]=\left[X_{2 j}, X_{2 n+1}\right]=0$.

\section{Generalisation of a classical result to the Heisenberg group}

We begin our investigation into the spectrum of sub-elliptic operators of the form (1.2) by working in the $n$-th Heisenberg group $\mathbb{U}^{n}$. The aim of this section is to prove a direct analogue of the following classical result for Schrödinger operators on $\mathbb{R}^{n}$ (see for example Theorem XIII.67 of [21]): consider the operator $H=\Delta+V$ on $\mathbb{R}^{n}$, where $\Delta$ is the standard Laplacian on $\mathbb{R}^{n}$ and $V \in L_{\text {loc }}^{1}\left(\mathbb{R}^{n}\right)$ is a potential which is bounded from below. Then, if $V(x) \rightarrow \infty$ as $|x| \rightarrow \infty$, it follows that $H$ has a purely discrete spectrum.

We will utilise a useful representation of the sub-Laplacian $\Delta_{\llbracket \llbracket n}$ on $\mathbb{T}^{n}$ given in [13]. Indeed, denote by $\widetilde{F}_{2 n+1}$ the partial Fourier transform with respect to the $(2 n+1)$-th variable:

$$
\mathcal{F}_{2 n+1} f(w, \zeta):=(2 \pi)^{-1 / 2} \int_{-\infty}^{\infty} e^{-i z \zeta} f(w, z) d z,
$$

for $(w, z) \in \mathbb{M}^{n}$. Then it can be shown that

$$
\widetilde{F}_{2 n+1}\left(-\Delta_{\mathbb{q}^{n}} f\right)(w, \zeta)=\sum_{j=1}^{n}\left(i \nabla_{\bar{w}_{j}}+\zeta \mathbf{A}\left(\bar{w}_{j}\right)\right)^{2} \widetilde{F}_{2 n+1} f(w, \zeta),
$$

where $w \in \mathbb{R}^{2 n}, \bar{w}_{j}=\left(w_{2 j-1}, w_{2 j}\right) \in \mathbb{R}^{2}$ for $j \in\{1, \ldots, n\}, \nabla_{\bar{w}_{j}}=\left(\partial_{w_{2 j-1}}, \partial_{w_{2 j}}\right)$ and $\mathbf{A}\left(\bar{w}_{j}\right)=\frac{1}{2}\left(-w_{2 j}, w_{2 j-1}\right)$. For fixed $\zeta$ and $j$ the operator $\left(i \nabla_{\bar{w}_{j}}+\zeta \mathbf{A}\left(\bar{w}_{j}\right)\right)^{2}$ has been well studied, since it corresponds to the Hamiltonian of a particle moving in a magnetic field (see [16]). Indeed, we have the following spectral decomposition:

$$
\mathcal{F}_{2 n+1}\left(-\Delta_{\llbracket \mathbb{n}} f\right)(w, \zeta)=\sum_{\mathbf{k} \in \mathbb{N}_{0}^{n}} \lambda_{\mathbf{k}}(\zeta) \mathcal{P}_{\mathbf{k}} \mathcal{F}_{2 n+1} f(w, \zeta), \quad w \in \mathbb{R}^{2 n}, \zeta \in \mathbb{R},
$$


where the eigenvalues are given by

$$
\lambda_{\mathbf{k}}(\zeta):=|\zeta|(2|\mathbf{k}|+n), \quad \mathbf{k} \in \mathbb{N}_{0}^{n},
$$

with $|\mathbf{k}|:=k_{1}+\cdots+k_{n}$, and $\mathcal{P}_{\mathbf{k}}$ are the corresponding the orthogonal eigenprojections. For $\mathbf{k} \in \mathbb{N}_{0}^{n}, \mathcal{P}_{\mathbf{k}}$ has an explicit kernel, and is given by

$$
\mathcal{P}_{\mathbf{k}} f(w)=\int_{\mathbb{R}^{2 n}} f(v) \bigotimes_{j=1}^{n} \pi_{k_{j}}\left(\bar{w}_{j}, \bar{v}_{j}\right) d \bar{v}_{j},
$$

where for $j \in\{1, \ldots, n\}$

$$
\pi_{k_{j}}\left(\bar{w}_{j}, \bar{v}_{j}\right)=\frac{|\zeta|}{2 \pi} e^{-\frac{|\zeta|}{2} i\left(w_{2 j-1} v_{2 j}-w_{2 j} v_{2 j-1}\right)-\frac{|\zeta|}{4}\left|\bar{w}_{j}-\bar{v}_{j}\right|^{2}} L_{k_{j}}\left(\frac{|\zeta|}{2}\left|\bar{w}_{j}-\bar{v}_{j}\right|^{2}\right)
$$

and $L_{k_{j}}$ is the $k_{j}$-th Laguerre polynomial. If we then define

$$
\hat{f}(\zeta, \mathbf{k}):=\left\|\mathcal{P}_{\mathbf{k}} \mathcal{F}_{2 n+1} f(\cdot, \zeta)\right\|_{L^{2}(d w)}
$$

for any $f \in L^{2}\left(\mathbb{H}^{n}\right)$, one can calculate that

$$
\int_{\mathbb{W}^{n}} f(x)\left(-\Delta_{\mathbb{\mathbb { A } ^ { n }}} f\right)(x) d x=\sum_{\mathbf{k} \in \mathbb{N}_{0}^{n}} \int_{\mathbb{R}}|\zeta|(2|\mathbf{k}|+n)|\hat{f}(\zeta, \mathbf{k})|^{2} d \zeta .
$$

Moreover, by the spectral theorem we can define a functional calculus for the operator $\Delta_{\mathbb{\square}} n$. Indeed, for any Borel function $\varphi:[0, \infty) \rightarrow \mathbb{R}$, we define

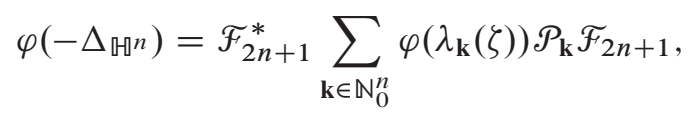

and then

$$
\int_{\mathbb{T}^{n}} f(x) \varphi\left(-\Delta_{\mathbb{\mathbb { T } ^ { n }}}\right) f(x) d x=\sum_{\mathbf{k} \in \mathbb{N}_{0}^{n}} \int_{\mathbb{R}} \varphi(|\zeta|(2|\mathbf{k}|+n))|\hat{f}(\zeta, \mathbf{k})|^{2} d \zeta .
$$

Finally, for a self-adjoint operator $H$ on $L^{2}\left(\mathbb{U}^{n}\right)$, let $\theta_{m}(H), m \in \mathbb{N} \cup\{0\}$ be as in the Min-Max principle i.e. either $\theta_{m}(H)$ is the $m$-th eigenvalue below the bottom of the essential spectrum of $H$, or $\theta_{m}(H)$ is the bottom of the essential spectrum and $\theta_{m}(H)=\theta_{m^{\prime}}(H)$ for $m^{\prime} \geq m$ (see for example Theorem XIII.1 of [21]).

Theorem 3.1. Suppose $V$ is in $L_{\text {loc }}^{1}\left(\mathbb{M}^{n}\right)$ and is bounded from below, and that $n \leq 2$. Suppose also that for every $L>0$ there exists $R_{L}>0$ such that $V(x) \geq L$ whenever $d(x) \geq R_{L}$. Then the operator $\mathscr{L}=-\Delta_{\mathbb{\square}} n+V$ on $L^{2}\left(\mathbb{U}^{n}\right)$ has empty essential spectrum. In particular it has a purely discrete set of eigenvalues and a complete set of eigenfunctions. 
Proof. Suppose $W$ is a bounded function, supported in a compact set $\Omega \subset \mathbb{R}^{2 n+1}$, so that

$$
\sup _{x \in \Omega}|W(x)| \leq M
$$

for some $M \in \mathbb{R}$. For $\varepsilon>0$ consider the operator

$$
W \varphi_{\varepsilon}\left(-\Delta_{\mathbb{G} \mathbb{q}^{n}}\right),
$$

where $\varphi_{\varepsilon}(t)=\left(\varepsilon t^{2}+t+1\right)^{-1}$ for $t \in \mathbb{R}$. Using the above observations, and following [13], we have that

$$
\begin{aligned}
\operatorname{Tr}\left(W^{2} \varphi_{\varepsilon}^{2}\left(-\Delta_{\mathbb{\square} n}\right)\right) \leq M^{2} \frac{1}{2 \pi} \sum_{\mathbf{k} \in \mathbb{N}_{0}^{n}} \int_{\Omega} \int_{-\infty}^{\infty} \varphi_{\varepsilon}^{2}\left(\lambda_{\mathbf{k}}(\zeta)\right) d \zeta \bigotimes_{j=1}^{n} \pi_{k_{j}}\left(\bar{w}_{j}, \bar{w}_{j}\right) d \bar{w}_{j} \\
=M^{2} \frac{|\Omega|}{(2 \pi)^{n+1}} \sum_{\mathbf{k} \in \mathbb{N}_{0}^{n}} \int_{-\infty}^{\infty} \varphi_{\varepsilon}^{2}\left(\lambda_{\mathbf{k}}(\zeta)\right)|\zeta|^{n} d \zeta \\
=M^{2} \frac{2|\Omega|}{(2 \pi)^{n+1}} \sum_{\mathbf{k} \in \mathbb{N}_{0}^{n}} \int_{0}^{\infty} \frac{|\zeta|^{n}}{\left(\varepsilon|\zeta|^{2}(2|\mathbf{k}|+n)^{2}+|\zeta|(2|\mathbf{k}|+n)+1\right)^{2}} d \zeta \\
\quad<\infty .
\end{aligned}
$$

Since $W \varphi_{\varepsilon}\left(-\Delta_{\mathbb{\llbracket} n}\right)$ is positive and self-adjoint on $L^{2}\left(\mathbb{H}^{n}\right)$, we thus have that $W \varphi_{\varepsilon}\left(-\Delta_{\mathbb{R} \mathbb{H}^{n}}\right)$ is Hilbert-Schmidt for all $\varepsilon>0$. Moreover,

$$
\left(\varepsilon \lambda_{\mathbf{k}}(\zeta)^{2}+\lambda_{\mathbf{k}}(\zeta)+1\right)^{-1} \rightarrow\left(\lambda_{\mathbf{k}}(\zeta)+1\right)^{-1} \equiv \varphi_{0}\left(\lambda_{\mathbf{k}}(\zeta)\right)
$$

in $L^{\infty}(\mathbb{R}) \times l^{\infty}\left(\mathbb{N}_{0}^{n}\right)$ as $\varepsilon \rightarrow 0$. Therefore $W \varphi_{0}\left(-\Delta_{\mathbb{Q}} n\right)$ is a norm-limit of HilbertSchmidt operators:

$$
\begin{aligned}
\left\|W\left(\varphi_{0}-\varphi_{\varepsilon}\right)\left(-\Delta_{\mathbb{\mathbb { T }}} n\right) \psi\right\|_{2}^{2} & \leq M^{2} \sum_{\mathbf{k} \in \mathbb{N}_{0}^{n}} \int_{\mathbb{R}}\left(\varphi_{0}-\varphi_{\varepsilon}\right)^{2}\left(\lambda_{\mathbf{k}}(\zeta)\right)|\hat{\psi}(\zeta, \mathbf{k})|^{2} d \zeta \\
& \leq \varepsilon^{2} M^{2} \sum_{\mathbf{k} \in \mathbb{N}_{0}^{n}} \int_{\mathbb{R}}|\hat{\psi}(\zeta, \mathbf{k})|^{2} d \zeta \\
& =\varepsilon^{2} M^{2}\|\psi\|_{2}^{2} .
\end{aligned}
$$

We can thus conclude that $W \varphi_{0}\left(-\Delta_{\mathbb{W}} n\right)$ is a compact operator, or in other words that $W$ is relatively compact with respect to $-\Delta_{\mathbb{H}^{n}}$.

Since $W \varphi_{0}\left(-\Delta_{\mathbb{H}^{n}}\right)$ is compact, by Weyl's Theorem (see Corollary 2 of Theorem XIII.14 of [21]),

$$
\sigma_{\mathrm{ess}}\left(-\Delta_{\mathbb{a} \mathbb{q}^{n}}+W\right)=\sigma_{\mathrm{ess}}\left(-\Delta_{\mathbb{q}} n\right)=[0, \infty) .
$$

Therefore by the Min-Max principle $\theta_{m}\left(-\Delta_{\mathbb{Q} \rrbracket^{n}}+W\right) \geq-1$ for $m$ sufficiently large. 
Now, given $a>0$, define $V_{a}$ by

$$
V_{a}(x)=\min \{V(x), a+1\}-a-1 .
$$

Then $V_{a}$ has compact support, since $V(x) \rightarrow \infty$ as $d(x) \rightarrow \infty$. Thus, by the above considerations, $\theta_{m}\left(-\Delta_{\mathbb{Q} \mathbb{Q}^{n}}+V_{a}\right) \geq-1$ for large $m$. Finally, since

$$
\theta_{m}(\mathscr{L}) \geq \theta_{m}\left(-\Delta_{\mathbb{q} n}+V_{a}\right)+a+1,
$$

we see that $\theta_{m}(\mathscr{L}) \geq a$ for large $m$. Since $a$ is arbitrary we reach the desired conclusion.

As mentioned in the introduction, we are interested in probability measures of the form $\mu_{U}(d x):=Z^{-1} e^{-U(x)} d x$ on $\mathbb{U}^{n}$, where $Z=\int_{\mathbb{Q}^{n}} e^{-U(x)} d x<\infty$, with which we can associate a positive and self-adjoint operator $\mathscr{L}=-\Delta_{\mathbb{q} n} n+\nabla_{\mathbb{\llbracket} n} U . \nabla_{\mathbb{q} n}$ on $L^{2}\left(d \mu_{U}\right)$.

Corollary 3.2. Suppose that $V=\frac{1}{4}\left|\nabla_{\mathbb{q}^{n}} U\right|^{2}-\frac{1}{2} \Delta_{\mathbb{Q}^{n}} U$ is in $L_{\mathrm{loc}}^{1}\left(\mathbb{\boxplus}^{n}\right)$, that $V$ is bounded from below, and that $V(x) \rightarrow \infty$ as $d(x) \rightarrow \infty$. Let $\mathscr{L}=-\Delta_{\mathbb{Q}^{n}}+$ $\nabla_{\mathbb{Q} n} U . \nabla_{\mathbb{Q} n}$, so that $\mathscr{L}$ is a positive self-adjoint operator on $L^{2}\left(\mu_{U}\right)$. Then

$$
\sigma_{\mathrm{ess}}(\mathscr{L})=\emptyset \text {. }
$$

Proof. This follows from Theorem 3.1 and the identity

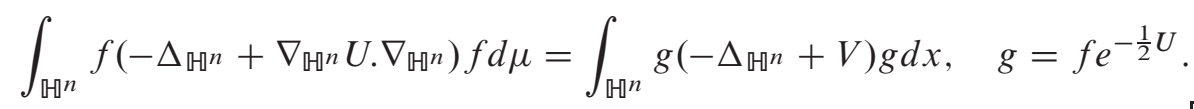

Following a recent trend (see [14] and references therein), for the rest of this paper we will concentrate on the case when $U$ is given as a power of one of the natural distance functions i.e. $U(x)=-\alpha d^{p}(x)$ or $U(x)=-\alpha N^{p}(x)$ with $p \in$ $(1, \infty)$ and $\alpha>0$, where $d$ and $N$ are the Carnot-Carathéodory and Kaplan distance functions respectively. We can therefore try and apply Corollary 3.2 in both these cases. However, this is not straightforward in either case. Indeed, when $U=\alpha d^{p}$ we have that

$$
\frac{1}{4}\left|\nabla_{\mathbb{R}^{n}} U\right|^{2}-\frac{1}{2} \Delta_{\mathbb{H}^{n}} U=\frac{\alpha^{2} p^{2}}{4} d^{2(p-1)}-\frac{\alpha p(p-1)}{2} d^{p-2}-\frac{\alpha p}{2} d^{p-1} \Delta_{\mathbb{H}^{n}} d,
$$

but we must understand this expression in the sense of distributions since $\Delta_{\mathbb{Q}}^{n} d$ is not defined on the centre of the group. Moreover, when $U=\alpha N^{p}$ we have

$$
\begin{aligned}
\frac{1}{4}\left|\nabla_{\mathbb{Q}^{n}} U\right|^{2}-\frac{1}{2} \Delta_{\mathbb{Q}^{n}} U= & \frac{\alpha^{2} p^{2}}{4} N^{2 p-4}(x)\|x\|^{2} \\
& -\left(\frac{\alpha p(p-1)}{2}+\frac{3 \alpha p}{2}\right) N^{p-4}(x)\|x\|^{2},
\end{aligned}
$$

for $x=(w, z) \in \mathbb{M}^{n}$, where $\|x\|=|w|$ as before, so that it is certainly not true that $V(x) \rightarrow \infty$ as $|x| \rightarrow \infty\left(V(x)=0\right.$ for all $\left.x=(0, z) \in \mathbb{U}^{n}\right)$. We therefore look for alternative methods to investigate these interesting cases. 


\section{Spectral information via functional inequalities}

The relationship between functional inequalities and the spectrum of operators is a very interesting and much studied one. Indeed, if $(\Omega, \mu)$ is a probability space and $(\mathscr{L}, \mathcal{D}(\mathscr{L}))$ is a positive self-adjoint operator on $L^{2}(\mu)$, then it is well-known that $\mathscr{L}$ has a gap at the bottom of its spectrum if and only if there exists a constant $c_{0}>0$ such that

$$
\mu(f-\mu(f))^{2} \leq c_{0} \&(f, f),
$$

where $(\mathcal{E}, \mathcal{D}(\mathscr{E}))$ is the Dirichlet form associated to $\mathscr{L}$. More recently this relationship has been further illustrated by the work of F. Cipriani [7] and F. Y. Wang [25] in which functional inequalities are introduced that characterise the essential spectra of operators under very general conditions. In this section we aim to use functional inequalities to overcome the problems encountered at the end of section 3 .

4.1. Super-Poincaré inequalities. To state the results of Wang and Cipriani in full generality, we first need the following two technical definitions.

Definition 4.1. A topological space $\Omega$ is a Lusin space if $\Omega$ is homeomorphic to a Borel subset of a compact metric space.

Remark 4.2. It should be noted that, as shown in Theorem 82.5 of [22], every complete metric space is a Lusin space. In particular, any H-type group $\mathbb{G}$ is a Lusin space.

Definition 4.3. Let $\Omega$ be a Lusin space, and $\mu$ a positive Radon measure on $\Omega$ having full topological support. A positive, self-adjoint operator $(\mathscr{L}, \mathscr{D}(\mathscr{L}))$ on $L^{2}(\mu)$, with associated closed Dirichlet form $(\mathcal{E}, \mathcal{D}(\mathscr{E}))$ defined by

$$
\mathcal{E}(f, g)=\mu(f \mathscr{L} g), \quad f, g \in \mathscr{D}(\mathscr{L}),
$$

is called a Persson operator if inf $\sigma_{\text {ess }}(\mathscr{L})=\sup \{\Sigma(K): K \subset \Omega$ is compact $\}$, where

$$
\Sigma(K):=\inf \left\{\frac{\mathscr{E}(f, f)}{\|f\|_{2}^{2}}: f \in \mathcal{D}(\mathcal{E}), \operatorname{supp}(f) \subset K^{c}\right\} .
$$

This class of operator was introduced by A. Persson in [20]. The result below is a combination of the independent work of Wang and Cipriani, and is explicitly stated in [26].

Theorem 4.4 (Wang/Cipriani). Let $\Omega$ be a Lusin space, $\mu$ a positive Radon measure on $\Omega$ having full topological support, and $(\mathscr{L}, \mathcal{D}(\mathscr{L}))$ a Persson operator on $L^{2}(\mu)$. Then the inequality

$$
\mu\left(f^{2}\right) \leq r \mu(f \mathscr{L} f)+\beta(r)(\mu|f|)^{2}, \quad \forall r>r_{0}, f \in \mathscr{D}(\mathscr{L}),
$$


for some decreasing function $\beta:\left(r_{0}, \infty\right) \rightarrow(0, \infty)$ and $r_{0} \geq 0$ holds if and only if $\sigma_{\mathrm{ess}}(\mathscr{L}) \subset\left[r_{0}^{-1}, \infty\right)$. In particular, (4.1) is satisfied with $\bar{r}_{0}=0$ if and only if $\sigma_{\mathrm{ess}}(\mathscr{L})=\emptyset$.

Inequality (4.1) is known as a super-Poincaré inequality. In a similar way to the generalisation of the standard logarithmic Sobolev inequality to the $L S_{q}$ inequality (see [5]), we can generalise the super-Poincaré inequality to a $q$-super-Poincaré inequality.

Definition 4.5. Let $(\Omega, \mu)$ be a probability space, equipped with a metric

$$
d: \Omega \times \Omega \rightarrow[0, \infty) .
$$

For $q \in(1,2]$, we say that $\mu$ satisfies a $q$-super-Poincaré inequality, or $\mathrm{SP}_{q}$ for short, with constant $r_{0}$, if

$$
\mu|f|^{q} \leq r \mu|\nabla f|^{q}+\beta(r)\left(\mu|f|^{\frac{q}{2}}\right)^{2} \quad \forall r>r_{0},
$$

for all locally Lipschitz functions $f$ and some $\beta:\left(r_{0}, \infty\right) \rightarrow(0, \infty)$, where

$$
|\nabla f|(x) \equiv \limsup _{d(x, y) \rightarrow 0} \frac{|f(x)-f(y)|}{d(x, y)} .
$$

Remark 4.6. In an H-type group $\mathbb{G}$ equipped with the Carnot-Carathéodory distance $d,\left|\nabla_{\mathbb{G}} f\right|(x)=\lim \sup _{d(x, y) \rightarrow 0}|f(x)-f(y)| / d(x, y)$.

4.2. Potentials defined by the Carnot-Carathéodory distance. Let $\mathbb{G}$ be an $\mathrm{H}$ type group as usual, equipped with the Carnot-Carathéodory distance $d$. Let $\mu_{p}$ be the probability measure on $\mathbb{G}$ defined by

$$
\mu_{p}(d x):=\frac{e^{-\alpha d^{p}(x)}}{Z} d x
$$

where $Z=\int e^{-\alpha d^{p}(x)} d x$ is the normalisation constant, and $p \in(1, \infty), \alpha>0$. Define

$$
\mathscr{L}_{p}:=-\Delta_{\mathbb{G}}+\nabla_{\mathbb{G}}\left(\alpha d^{p}\right) \cdot \nabla_{\mathbb{G}}=-\Delta_{\mathbb{G}}+\alpha p d^{p-1} \nabla_{\mathbb{G}} d \cdot \nabla_{\mathbb{G}}
$$

as a positive self-adjoint operator acting on $L^{2}\left(\mu_{p}\right)$. The associated Dirichlet form $\varepsilon_{p}(f, g)$ is then given by

$$
\mathcal{E}_{p}(f, g)=\mu_{p}\left(f \mathscr{L}_{p} g\right)=\int_{\mathbb{G}} \nabla_{\mathbb{G}} f \cdot \nabla_{\mathbb{G}} g d \mu_{p} .
$$

We aim to prove the following: 
Theorem 4.7. For any $p>1$ the positive self-adjoint operator $\mathscr{L}_{p}$ on $L^{2}\left(\mu_{p}\right)$ given by (4.4) has a purely discrete spectrum i.e. $\sigma_{\mathrm{ess}}\left(\mathscr{L}_{p}\right)=\emptyset$.

The idea is to use Theorem 4.4. It is clear that we first need to show that $\mathscr{L}_{p}$ is a Persson operator. We make use of the following very general result stated in the setting of Dirichlet forms, proved by G. Grillo in [12] (and also stated explicitly in [7]).

Theorem 4.8 (Grillo). Let $(\Omega, \mu)$ be a locally compact, separable metric space, and $(\mathscr{E}, \mathcal{D}(\mathcal{E}))$ a regular, strongly local Dirichlet form on $L^{2}(\Omega)$, with associated positive self-adjoint operator $(\mathscr{L}, \mathcal{D}(\mathscr{L}))$.

Define the associated intrinsic pseudo-metric $\rho$ on $\Omega$ by

$$
\rho(x, y):=\sup \left\{|f(x)-f(y)|: f \in \mathcal{D}(\mathcal{E}) \cap C_{0}(\Omega), \Gamma(f, f) \leq 1\right\}
$$

where for $f \in \mathcal{D}(\mathcal{E}), \Gamma(f, f)$ is such that

$$
\int_{\Omega} g \Gamma(f, f) d \mu=\mathcal{E}(g f, f)-\frac{1}{2} \varepsilon\left(f^{2}, g\right), \quad f, g \in \mathcal{D}(\mathscr{E}) \cap C_{0}(\Omega) .
$$

Suppose $\rho$ is a true metric generating the original topology of $\Omega$. Then the operator $(\mathscr{L}, \mathcal{D}(\mathscr{L}))$ is a Persson operator.

Corollary 4.9. The operator $\mathscr{L}_{p}$ given by (4.4) acting on $L^{2}\left(\mu_{p}\right)$ is a Persson operator.

Proof. The intrinsic pseudo-metric associated to $\mathscr{L}_{p}$ is given by

$$
\rho(x, y)=\sup \left\{|f(x)-f(y)|:\left|\nabla_{\mathbb{G}} f\right|^{2} \leq 1\right\},
$$

which is nothing more than the Carnot-Carathéodory distance (by definition).

The next result we prove on route to Theorem 4.7 is that the measures $\mu_{p}$ satisfy certain super-Poincaré inequalities.

Theorem 4.10. Let $\mu_{p}$ be the probability measure on $\mathbb{G}$ given by (4.3). (i) Suppose $p \geq 2$. Then $\mu_{p}$ satisfies an $\mathrm{SP}_{q}$ inequality with constant $r_{0}=0$ i.e.

$$
\mu_{p}|f|^{q} \leq r \mu_{p}\left|\nabla_{\mathbb{G}} f\right|^{q}+\beta(r)\left(\mu_{p}|f|^{\frac{q}{2}}\right)^{2}, \quad \forall r>0,
$$

where $\frac{1}{p}+\frac{1}{q}=1$, for some function $\beta:(0, \infty) \rightarrow(0, \infty)$ and for all locally Lipschitz functions $f$. i.e.

(ii) Suppose $p \in(1,2]$. Then $\mu_{p}$ satisfies an $\mathrm{SP}_{2}$ inequality with constant $r_{0}=0$

$$
\mu_{p}\left(f^{2}\right) \leq r \mu_{p}\left|\nabla_{\mathbb{G}} f\right|^{2}+\beta(r)\left(\mu_{p}|f|\right)^{2}, \quad \forall r>0,
$$

for some function $\beta:(0, \infty) \rightarrow(0, \infty)$ and for all locally Lipschitz functions $f$. 
Proof. The idea is to pass from a logarithmic Sobolev inequality for the measure $\mu_{p}$, which is true by Theorem 4.1 of [14], to a super-Poincaré inequality by adapting the methods of [25].

We first deal with the case $p \geq 2$. Without loss of generality we may assume that $f \geq 0$. By Theorem 4.1 of [14], we have that there exists a constant $c$ such that

$$
\mu_{p}\left(f^{q} \log \frac{f^{q}}{\mu_{p} f^{q}}\right) \leq c \mu_{p}\left|\nabla_{\mathbb{G}} f\right|^{q}
$$

where $\frac{1}{p}+\frac{1}{q}=1$. Let $g:(0, \infty) \rightarrow \mathbb{R}$ be given by $g(\xi)=t \xi-\xi \log \left(\frac{\xi^{2}}{a}\right)$ for any $t, a>0$. We then have that

$$
\max _{\{\xi>0\}} g(\xi)=2 \sqrt{a e^{t-2}}
$$

Suppose that $\mu_{p}\left(f^{\frac{q}{2}}\right)=1$, and set $a=\mu_{p}\left(f^{q}\right)$. Then by (4.7), for all $t>0$,

$$
\begin{aligned}
& t f^{\frac{q}{2}}-f^{\frac{q}{2}} \log \left(\frac{f^{q}}{a}\right) \leq 2 \sqrt{a e^{t-2}} \\
& \Rightarrow \mu_{p}\left(f^{q} \log \frac{f^{q}}{a}\right) \geq t a-2 \sqrt{a e^{t-2}},
\end{aligned}
$$

using the fact that $f \geq 0$ and $\mu_{p}\left(f^{\frac{q}{2}}\right)=1$. Setting $b=\mu_{p}\left|\nabla_{\mathbb{G}} f\right|^{q}$, by (4.6), we then have

$$
t a-2 \sqrt{a e^{t-2}}-c b \leq 0
$$

Solving this quadratic inequality gives

$$
\sqrt{a} \leq \frac{2 \sqrt{e^{t-2}}}{2 t}+\frac{\sqrt{4 e^{t-2}+4 t c b}}{2 t}
$$

for $t>0$, so that $a \leq \frac{2 c}{t} b+4 \frac{e^{t-2}}{t^{2}}$. Thus

$$
\mu_{p}\left(f^{q}\right) \leq \frac{2 c}{t} \mu_{p}\left|\nabla_{\mathbb{G}} f\right|^{q}+4 \frac{e^{t-2}}{t^{2}}
$$

for all $t>0$ and $f$ such that $\mu_{p}\left(f^{\frac{q}{2}}\right)=1$. Replacing $f$ by $\frac{f}{\mu_{p}\left(f^{q / 2}\right)^{2 / q}}$ yields

$$
\mu_{p}\left(f^{q}\right) \leq \frac{2 c}{t} \mu_{p}\left|\nabla_{\mathbb{G}} f\right|^{q}+4 \frac{e^{t-2}}{t^{2}}\left(\mu_{p}\left(f^{\frac{q}{2}}\right)\right)^{2}
$$

for all $t>0$. Taking $r=\frac{2 c}{t}$ we see that $\mathrm{SP}_{q}$ holds, proving part (i).

In the case where $p \in(1,2)$, we no longer have an inequality of the type (4.6). However, by Theorem 4.3 of [14] there exists a constant $c \in(0, \infty)$ such that

$$
\mu_{p}\left(f^{2}\left[\log \left(1+f^{2}\right)\right]^{\theta}\right) \leq c \mu_{p}\left|\nabla_{\mathbb{G}} f\right|^{2}+(\log 2)^{\theta}, \quad \mu_{p}\left(f^{2}\right)=1,
$$


where $\theta=\frac{2(p-1)}{p}$. In this case we instead let $g:(0, \infty) \rightarrow \mathbb{R}$ be given by $g(\xi)=t \xi-$ $\xi\left[\log \left(1+\frac{\xi^{2}}{a}\right)\right]^{\theta}$ for $t, a>0$, so that $\sup _{\{\xi>0\}} g(\xi) \leq t \sqrt{a\left(e^{t^{1 / \theta}}-1\right)}$. Proceeding now in a very similar way as in the proof of part (i), we arrive at an $\mathrm{SP}_{2}$ inequality.

The final result we need is that $\mathrm{SP}_{q}$ inequalities are stronger than $\mathrm{SP}_{2}$ inequalities (at least when the dimension of the underlying space is finite).

Lemma 4.11. Suppose an arbitrary probability measure $\mu$ on $\mathbb{G}$ satisfies an $\mathrm{SP}_{q}$ inequality with $q \in(1,2]$ and constant $r_{0}=0$. Then $\mu$ also satisfies an $\mathrm{SP}_{2}$ inequality with constant $r_{0}=0$.

Proof. As usual, without loss of generality we may suppose $f \geq 0$. Let $q<2$ (there is nothing to prove if $q=2$ ). Applying the $\mathrm{SP}_{q}$ inequality to $f^{\frac{2}{q}}$ yields,

$$
\mu\left(f^{2}\right) \leq r \mu\left|\nabla_{\mathbb{G}} f^{\frac{2}{q}}\right|^{q}+\beta(r)(\mu f)^{2}, \quad \forall r>0 .
$$

Therefore for all $r>0$, we have by Hölder's inequality followed by Young's inequality,

$$
\begin{aligned}
\mu\left(f^{2}\right) & \leq \frac{2^{q} r}{q^{q}} \mu\left(f^{2-q}\left|\nabla_{\mathbb{G}} f\right|^{q}\right)+\beta(r)(\mu f)^{2} \\
& \leq \frac{2^{q-1} r}{q^{q-1}} \tau^{\frac{2-q}{q}} \mu\left|\nabla_{\mathbb{G}} f\right|^{2}+\frac{2^{q-1} r(2-q)}{q^{q}} \tau^{-1} \mu\left(f^{2}\right)+\beta(r)(\mu f)^{2}
\end{aligned}
$$

for all $\tau>0$. Taking $\tau=\frac{2^{q} r(2-q)}{q^{q}}$ we see that

$$
\begin{aligned}
\frac{1}{2} \mu\left(f^{2}\right) & \leq \frac{2^{q-1} r}{q^{q-1}} \tau^{\frac{2-q}{q}} \mu\left|\nabla_{\mathbb{G}} f\right|^{2}+\beta(r)(\mu f)^{2} \\
& =\frac{2^{q-1}}{q^{q-1}}\left(\frac{2}{q}\right)^{2-q}(2-q)^{\frac{2-q}{q}} r^{\frac{2}{q}} \mu\left|\nabla_{\mathbb{G}} f\right|^{2}+\beta(r)(\mu f)^{2} \\
\Longrightarrow \mu\left(f^{2}\right) & \leq \frac{4}{q}(2-q)^{\frac{2-q}{q}} r^{\frac{2}{q}} \mu\left|\nabla_{\mathbb{G}} f\right|^{2}+2 \beta(r)(\mu f)^{2} .
\end{aligned}
$$

Taking $s=\frac{4}{q}(2-q)^{\frac{2-q}{q}} r^{\frac{2}{q}}$ then yields the result.

Proof of Theorem 4.7. We can now combine all of the above results to arrive at Theorem 4.7. Indeed by Theorem 4.10 and Lemma 4.11, we have that the measures $\mu_{p}$ satisfy a super-Poincaré inequality with constant $r_{0}=0$ for all $p>1$. Moreover, by Corollary $4.9, \mathscr{L}_{p}$ is a Persson operator, so that we may conclude by applying Theorem 4.4. 
Remark 4.12. Since the main result we use to prove Theorem 4.7 is that the measure $\mu_{p}$ satisfies a logarithmic Sobolev inequality, we can greatly extend the class of measures for which these results remain valid when $p \geq 2$. Indeed, if we define

$$
\tilde{\mu}_{p}=\frac{e^{-W-V}}{Z^{\prime}} d \mu_{p}
$$

with $W$ a differentiable potential satisfying $\left|\nabla_{\mathbb{G}} W\right|^{q} \leq d^{\gamma}+K$ for constants $K$ and $\gamma \in(0, p)$, and $V$ a measurable function such that $\max V-\min V<\infty$, then all the above results remain true for $\tilde{\mu}_{p}$. This follows from Corollary 4.1 of [14].

Corollary 4.13. Let $\rho_{t}(x, y)$ be the heat kernel at time $t$ on an H-type group $\mathbb{G}$ i.e. $\rho_{t}(x, y)$ is the function (smooth by Hörmander's theorem) such that

$$
e^{t \Delta_{\mathbb{G}}} f(x)=\int_{\mathbb{G}} \rho_{t}(x, y) f(y) d y .
$$

Let $\rho(x):=\rho_{1}(x, e)$ and define

$$
\mathscr{L}_{H}:=-\Delta_{\mathbb{G}}+\nabla_{\mathbb{G}} \log \rho \cdot \nabla_{\mathbb{G}} .
$$

Then $\mathscr{L}_{H}$ is a positive self-adjoint operator on $L^{2}\left(\mu_{H}\right)$, where $\mu_{H}(d x):=\rho(x) d x$, and $\sigma_{\mathrm{ess}}\left(\mathscr{L}_{H}\right)=\emptyset$, so that $\mathscr{L}_{H}$ has a purely discrete spectrum.

Remark 4.14. $\mathscr{L}_{H}$ can be regarded as the natural Ornstein-Uhlenbeck generator on $\mathbb{G}$, as suggested in [3], and the resulting Markov process is the natural OU-process associated to the hypoelliptic diffusion on $\mathbb{G}$.

Proof. It follows exactly as above, once we have recalled that $\mu_{H}$ satisfies a logarithmic Sobolev inequality (see [1], [14] and [17]).

4.3. Potentials defined by the Kaplan distance. An interesting question to ask is whether one can replace the Carnot-Carathéodory distance with the Kaplan distance in the above work. At first glance such a question might seem simple, since all homogeneous metrics on $\mathbb{G}$ are equivalent. However, as we will see, this is not the case, and there are some fundamental differences between the two situations which arise from the different smoothness properties of the two distance functions.

To proceed, suppose now that we are working in an H-type group $\mathbb{G}$ as before, but now equipped with a probability measure and associated self-adjoint operator given by

$$
v_{p}(d x):=\frac{e^{-\alpha N^{p}(x)}}{Z} d x, \quad \mathcal{T}_{p}=-\Delta_{\mathbb{G}}+\alpha p N^{p-1} \nabla_{\mathbb{G}} N \cdot \nabla_{\mathbb{G}}
$$

respectively, where $p \in(1, \infty), \alpha>0$ and $Z=\int e^{-\alpha N^{p}(x)} d x$.

Trying to apply the functional inequality approach of section 4.1 , we immediately come up against a problem in the form of Theorem 6.3 from [14]: 
Theorem 4.15 (Hebisch-Zegarliński). The measure $v_{p}$ on $\mathbb{G}$ given by (4.10) with $p>1$ satisfies no $L S_{q}$ inequality with $q \in(1,2]$.

Thus we cannot simply follow the proof of Theorem 4.7 to conclude that the operator $\mathcal{T}_{p}$ given by (4.10) has empty essential spectrum. Theorem 4.15 illustrates a major difference in the behaviour of the measures defined with the Carnot-Carathéodory distance and those defined with the Kaplan distance.

In view of this, it seems that the problem of gaining spectral information about the operator $\mathcal{T}_{p}$ given by (4.10) is an interesting one. We therefore start by asking whether such operators have a spectral gap. This question is completely answered by Theorems 4.16 and 4.19 below.

Theorem 4.16. If $p<2$, then the measure $v_{p}$ given by (4.10) does not satisfy a spectral gap inequality. In particular the operator $\mathcal{T}_{p}$ given by (4.10) does not have a spectral gap, and hence it does not have empty essential spectrum.

To prove this, we make use of the following result, quoted from [14] (Lemma 6.3), and we refer the reader to this work for the proof.

Lemma 4.17. Let $f$ be a smooth function on $\mathbb{G}$ and $d$ the Carnot-Carathéodory distance as usual. Then at points $x_{0} \in \mathbb{G}$ such that $\left(\nabla_{\mathbb{G}} f\right)\left(x_{0}\right)=0$ we have

$$
\left|f(x)-f\left(x_{0}\right)\right| \leq O\left(d^{2}\left(x, x_{0}\right)\right)
$$

for all $x \in \mathbb{G}$.

Proof of Theorem 4.16. Let $p<2$ and suppose for a contradiction that there exists a constant $c_{0}$ such that

$$
v_{p}\left(f^{2}\right)-\left(v_{p} f\right)^{2} \leq c_{0} v_{p}\left|\nabla_{\mathbb{G}} f\right|^{2}
$$

for all locally Lipschitz functions $f$.

Fix $x_{0}=(0, z) \in \mathbb{G}$ for $z \in \mathbb{R}^{m} \backslash\{0\}$. Then $\left|\nabla_{\mathbb{G}} N\left(x_{0}\right)\right|=\frac{\left\|x_{0}\right\|}{N\left(x_{0}\right)}=0$ by Proposition 2.7, so that $\nabla_{\mathbb{G}} N\left(x_{0}\right)=0$. Similarly $\nabla_{\mathbb{G}} N\left(-x_{0}\right)=0$. Let $r_{0}>0$ be small enough so that $0 \notin B_{r_{0}}\left(x_{0}\right)=\left\{y^{\prime} \in \mathbb{G}: d\left(y^{\prime}, x_{0}\right) \leq r_{0}\right\}$. Then $N$ is smooth on $B_{r_{0}}\left(x_{0}\right)$, and by Lemma 4.17 there exists a constant $C_{1}$ such that

$$
\left|N(y)-N\left(x_{0}\right)\right| \leq C_{1} r_{0}^{2},
$$

for all $y \in B_{r_{0}}\left(x_{0}\right)$. The same holds for $y \in B_{r_{0}}\left(-x_{0}\right)$. We now dilate by a factor of $t>0$. Since $N$ is homogeneous, we have that

$$
\left|N(y)-N\left(\delta_{t}\left(x_{0}\right)\right)\right|=t\left|N\left(\delta_{t-1}(y)\right)-N\left(x_{0}\right)\right| \leq C_{1} t r_{0}^{2}
$$

for $\delta_{t-1}(y) \in B_{r_{0}}\left(x_{0}\right) \Leftrightarrow y \in B_{t r_{0}}\left(\delta_{t}\left(x_{0}\right)\right)$, where $\left(\delta_{t}\right)_{t>0}$ is the natural family of dilations given by Definition 2.3. The same holds for $y \in B_{t r_{0}}\left(\delta_{t}\left(-x_{0}\right)\right)$. 
Let $r=t r_{0}$. We have for $y \in B_{r}\left(\delta_{t}\left(x_{0}\right)\right)$ or $y \in B_{r}\left(\delta_{t}\left(-x_{0}\right)\right)$

$$
\begin{aligned}
\left|N^{p}(y)-N^{p}\left(\delta_{t}\left(x_{0}\right)\right)\right| & \leq C_{2} N^{p-1}\left(\delta_{t}\left(x_{0}\right)\right)\left|N(y)-N\left(\delta_{t}\left(x_{0}\right)\right)\right| \\
& \leq C_{3} t^{p-1} t r_{0}^{2}=C_{3} t^{p} r_{0}^{2}
\end{aligned}
$$

for some constants $C_{2}, C_{3}$, using the mean value theorem. Thus if we take $t$ large enough so that $r_{0}=t^{-\frac{p}{2}}$, we have

$$
\left|N^{p}(y)-N^{p}\left(\delta_{t}\left(x_{0}\right)\right)\right| \leq C_{3}, \quad \forall y \in B_{r}\left(\delta_{t}\left(x_{0}\right)\right) \cup B_{r}\left(\delta_{t}\left(-x_{0}\right)\right),
$$

so that

$$
\left|\frac{e^{-\beta N^{p}(y)}}{e^{-\beta N^{p}\left(\delta_{t}\left(x_{0}\right)\right)}}\right| \approx 1
$$

for all $y \in B_{r}\left(\delta_{t}\left(x_{0}\right)\right) \cup B_{r}\left(\delta_{t}\left(-x_{0}\right)\right)$. Now define

$$
\begin{aligned}
\varphi(y)= & \max \left\{\min \left\{2-\frac{N\left(y, \delta_{t}\left(x_{0}\right)\right)}{r}, 1\right\}, 0\right\} \\
& -\max \left\{\min \left\{2-\frac{N\left(y, \delta_{t}\left(-x_{0}\right)\right)}{r}, 1\right\}, 0\right\} .
\end{aligned}
$$

Then $\varphi$ is a Lipschitz function supported on balls of radius $r$ centred at $\delta_{t}\left(x_{0}\right)$ and $\delta_{t}\left(-x_{0}\right)$, which is equal to 1 on balls of radius $r / 2$ around these two points and decays to zero linearly in between $r / 2$ and $r$. We can note that by construction, and since the measure $v_{p}$ is symmetric about the origin,

$$
\int_{\mathbb{G}} \varphi(y) d v_{p}(y)=0 .
$$

Applying the spectral gap inequality (4.11) to the function $\varphi$, then yields

$$
\begin{aligned}
& \int_{B_{r}\left(\delta_{t}\left(x_{0}\right)\right) \cup B_{r}\left(\delta_{t}\left(-x_{0}\right)\right)} \varphi^{2}(y) d v_{p}(y) \\
& \quad \leq c_{0} \int_{B_{r}\left(\delta_{t}\left(x_{0}\right)\right) \cup B_{r}\left(\delta_{t}\left(-x_{0}\right)\right)}\left|\nabla_{\mathbb{G}} \varphi(y)\right|^{2} d v_{p}(y) .
\end{aligned}
$$

Now, using (4.13), there exist positive constants $C_{4}$ and $C_{5}$ such that

$$
\begin{aligned}
& \int_{B_{r}\left(\delta_{t}\left(x_{0}\right)\right) \cup B_{r}\left(\delta_{t}\left(-x_{0}\right)\right)} \varphi^{2}(y) d v_{p}(y) \\
& \quad \geq 2 \int_{B_{\frac{r}{2}}\left(\delta_{t}\left(x_{0}\right)\right)} d v_{p}(y) \geq C_{4} r^{Q} e^{-\beta t^{p} N^{p}\left(x_{0}\right)},
\end{aligned}
$$

and

$$
\begin{aligned}
& \int_{B_{r}\left(\delta_{t}\left(x_{0}\right)\right) \cup B_{r}\left(\delta_{t}\left(-x_{0}\right)\right)}\left|\nabla_{\mathbb{G}} \varphi(y)\right|^{2} d v_{p}(y) \\
& \quad \leq 2 r^{-2} \int_{B_{r}\left(\delta_{t}\left(x_{0}\right)\right)} d v_{p}(y) \leq C_{5} r^{-2+Q} e^{-\beta t^{p} N^{p}\left(x_{0}\right)},
\end{aligned}
$$


where $Q=n+2 m$ is the homogeneous dimension of the group. Using these estimates in (4.15) yields

$$
C_{4} \leq c_{0} C_{5} r^{-2}
$$

where $r=t r_{0}=t^{1-\frac{p}{2}}$. Since $p<2$ and $t$ can be taken arbitrarily large, this is a contradiction.

Remark 4.18. Theorem 4.16 provides another illustration of a fundamental difference between the operators $\mathscr{L}_{p}$ defined by (4.4) with the Carnot-Carathéodory distance and the operators $\mathcal{T}_{p}$ defined by (4.10) with the Kaplan distance. Indeed, with $p \in(1,2)$, by Theorem $4.7, \mathscr{L}_{p}$ has empty essential spectrum, while $\mathcal{T}_{p}$ does not even have a spectral gap.

Theorem 4.19. If $p \geq 2$, the measure $v_{p}$ given by (4.10) satisfies a $q$-spectral gap inequality, i.e. there exists a constant $c_{0}$ such that

$$
v_{p}\left|f-v_{p} f\right|^{q} \leq c_{0} v_{p}\left|\nabla_{\mathbb{G}} f\right|^{q}
$$

for all locally Lipschitz functions $f$, where $\frac{1}{q}+\frac{1}{p}=1$. In particular, for $p \geq 2$ the operator $\mathcal{T}_{p}$ associated to $v_{p}$ given by (4.10) has a spectral gap.

To prove this we adapt the methods of Hebisch and Zegarliński in [14], and proceed through an intermediate inequality which is similar to the $U$-bound studied there.

Lemma 4.20. For $p \geq 2$ there exist constants $A, B$ such that

$$
v_{p}\left(|f|^{q} N^{p-2}\|\cdot\|^{2}\right) \leq A v_{p}\left|\nabla_{\mathbb{G}} f\right|^{q}+B v_{p}|f|^{q}
$$

for all locally Lipschitz functions $f$, where $\frac{1}{p}+\frac{1}{q}=1$, and $\|x\|=|w|$ for $x=$ $(w, z) \in \mathbb{G}=\mathbb{R}^{n} \times \mathbb{R}^{m}$.

Proof. We can suppose that $f \geq 0$ and moreover that $f \in C_{0}^{\infty}(\mathbb{G})$. Indeed, for general $f \in C_{0}^{\infty}(\mathbb{G})$ we can write $|f|=f^{+}+f^{-}$and apply the inequality to each component separately. For general locally Lipschitz functions $f$, one can take a sequence $\left\{f_{n}\right\} \subset C_{0}^{\infty}(\mathbb{G})$ satisfying (4.16) and converging to $f$ in the uniform topology. Taking the limit as $n \rightarrow \infty$ then yields the inequality for $f$.

For such $f$, note that

$$
\left(\nabla_{\mathbb{G}} f\right) e^{-\alpha N^{p}}=\nabla_{\mathbb{G}}\left(f e^{-\alpha N^{p}}\right)+\alpha p f N^{p-1}\left(\nabla_{\mathbb{G}} N\right) e^{-\alpha N^{p}} .
$$

We now take the inner product of both sides of this equation with $\frac{N}{\|x\|} \nabla_{\mathbb{G}} N$ and integrate over $\mathbb{G}$ to arrive at

$$
\begin{aligned}
\int \frac{N}{\|x\|} \nabla_{\mathbb{G}} N \cdot\left(\nabla_{\mathbb{G}} f\right) e^{-\alpha N^{p}} d x= & \int \frac{N}{\|x\|} \nabla_{\mathbb{G}} N \cdot \nabla_{\mathbb{G}}\left(f e^{-\alpha N^{p}}\right) d x \\
& +\alpha p \int f \frac{N^{p}}{\|x\|}\left|\nabla_{\mathbb{G}} N\right|^{2} e^{-\alpha N^{p}} d x .
\end{aligned}
$$


By the Cauchy-Schwarz inequality, we then have

$$
\begin{aligned}
& \int \frac{N}{\|x\|}\left|\nabla_{\mathbb{G}} N\right|\left|\nabla_{\mathbb{G}} f\right| e^{-\alpha N^{p}} d x \\
& \geq \int \frac{N}{\|x\|} \nabla_{\mathbb{G}} N \cdot \nabla_{\mathbb{G}}\left(f e^{-\alpha N^{p}}\right) d x+\alpha p \int f \frac{N^{p}}{\|x\|}\left|\nabla_{\mathbb{G}} N\right|^{2} e^{-\alpha N^{p}} d x,
\end{aligned}
$$

so that by Proposition 2.7 and integration by parts,

$$
\begin{aligned}
& \int\left|\nabla_{\mathbb{G}} f\right| e^{-\alpha N^{p}} d x \\
& \geq \int \frac{N}{\|x\|} \nabla_{\mathbb{G}} N \cdot \nabla_{\mathbb{G}}\left(f e^{-\alpha N^{p}}\right) d x+\alpha p \int f N^{p-2}\|x\| e^{-\alpha N^{p}} d x \\
& =-\int f \nabla_{\mathbb{G}} \cdot\left(\frac{N}{\|x\|} \nabla_{\mathbb{G}} N\right) e^{-\alpha N^{p}} d x+\alpha p \int f N^{p-2}\|x\| e^{-\alpha N^{p}} d x .
\end{aligned}
$$

Note that

$$
\begin{aligned}
\nabla_{\mathbb{G}} \cdot\left(\frac{N}{\|x\|} \nabla_{\mathbb{G}} N\right) & =\frac{\left|\nabla_{\mathbb{G}} N\right|^{2}}{\|x\|}+\frac{N}{\|x\|} \Delta_{\mathbb{G}} N-\frac{N}{\|x\|^{2}} \nabla_{\mathbb{G}} N \cdot \nabla_{\mathbb{G}}\|x\| \\
& =\frac{\|x\|}{N^{2}}+(Q-1) \frac{\|x\|}{N^{2}}-\frac{N}{\|x\|^{2}} \nabla_{\mathbb{G}} N \cdot \nabla_{\mathbb{G}}\|x\| .
\end{aligned}
$$

Moreover, denoting $x=(w, z) \in \mathbb{G}$ and recalling the results from Section 2, it can be calculated that

$$
\nabla_{\mathbb{G}} N \cdot \nabla_{\mathbb{G}}\|x\|=\frac{\|x\|^{3}}{N^{3}} .
$$

Using this in (4.18) yields

$$
\nabla_{\mathbb{G}} \cdot\left(\frac{N}{\|x\|} \nabla_{\mathbb{G}} N\right)=(Q-1) \frac{\|x\|}{N^{2}} .
$$

Putting (4.19) in (4.17) then gives

$$
\alpha p v_{p}\left(f N^{p-2}\|\cdot\|\right) \leq v_{p}\left|\nabla_{\mathbb{G}} f\right|+(Q-1) v_{p}\left(f \frac{\|\cdot\|}{N^{2}}\right) .
$$

Replacing $f$ by $f\|\cdot\|$, we see that

$$
\alpha p v_{p}\left(f N^{p-2}\|\cdot\|^{2}\right) \leq v_{p}\left(\|\cdot\|\left|\nabla_{\mathbb{G}} f\right|\right)+Q v_{p}(f),
$$

using the fact that $\|x\| \leq N(x)$ and $\left|\nabla_{\mathbb{G}}\|x\|\right|=1$. Now, by replacing $f$ by $f^{q}$ with $\frac{1}{q}+\frac{1}{p}=1$ in (4.20), we then arrive at

$$
\begin{aligned}
\alpha p v_{p}\left(f^{q} N^{p-2}\|\cdot\|^{2}\right) & \leq q v_{p}\left(\|\cdot\| f^{q-1}\left|\nabla_{\mathbb{G}} f\right|\right)+Q v_{p}\left(f^{q}\right) \\
& \leq \frac{1}{\varepsilon^{q-1}} v_{p}\left|\nabla_{\mathbb{G}} f\right|^{q}+\frac{q}{p} \varepsilon v_{p}\left(\|\cdot\|^{p} f^{q}\right)+Q v_{p}\left(f^{q}\right),
\end{aligned}
$$


for all $\varepsilon>0$, using Young's inequality. Thus

$$
\alpha p v_{p}\left(f^{q} N^{p-2}\|\cdot\|^{2}\right) \leq \frac{1}{\varepsilon^{q-1}} v_{p}\left|\nabla_{\mathbb{G}} f\right|^{q}+\frac{q}{p} \varepsilon v_{p}\left(N^{p-2}\|\cdot\|^{2} f^{q}\right)+Q v_{p}\left(f^{q}\right),
$$

so that, by taking $\varepsilon<\frac{p^{2}}{q} \alpha$, we see that

$$
v_{p}\left(f^{q} N^{p-2}\|\cdot\|^{2}\right) \leq A v_{p}\left|\nabla_{\mathbb{G}} f\right|^{q}+B v_{p}\left(f^{q}\right),
$$

with $A=\varepsilon^{-q+1}\left(\alpha p-\frac{q}{p} \varepsilon\right)^{-1}$, and $B=Q\left(\alpha p-\frac{q}{p} \varepsilon\right)^{-1}$.

We are now in a position to prove Theorem 4.19.

Proof of Theorem 4.19. First note that

$$
v_{p}\left|f-v_{p} f\right|^{q} \leq 2^{q} v_{p}|f-m|^{q},
$$

for all $m \in \mathbb{R}$. Now, for $R>0$ and $L>1$,

$$
\begin{aligned}
v_{p}|f-m|^{q}= & v_{p}\left(|f-m|^{q} \mathbf{1}_{\left\{\|\cdot\|^{2} N^{p-2} \geq R\right\}}\right) \\
& +v_{p}\left(|f-m|^{q} \mathbf{1}_{\left\{\|\cdot\|^{2} N^{p-2} \leq R\right\}} \mathbf{1}_{\{N \leq L\}}\right) \\
& +v_{p}\left(|f-m|^{q} \mathbf{1}_{\left\{\|\cdot\|^{2} N^{p-2} \leq R\right\}} \mathbf{1}_{\{N \geq L\}}\right) .
\end{aligned}
$$

We treat each of the three terms of (4.22) separately.

First term of (4.22): This can be estimated using Lemma 4.20. Indeed

$$
\begin{aligned}
v_{p}\left(|f-m|^{q} \mathbf{1}_{\left\{\|\cdot\|^{2} N^{p-2} \geq R\right\}}\right) & \leq \frac{1}{R} v_{p}\left(|f-m|^{q} N^{p-2}\|\cdot\|^{2}\right) \\
& \leq \frac{A}{R} v_{p}\left|\nabla_{\mathbb{G}} f\right|^{q}+\frac{B}{R} v_{p}|f-m|^{q} .
\end{aligned}
$$

Second term of (4.22): We have

$$
\begin{aligned}
v_{p}\left(|f-m|^{q} \mathbf{1}_{\left\{\|\cdot\|^{2} N^{p-2} \leq R\right\}} \mathbf{1}_{\{N \leq L\}}\right) & \leq v_{p}\left(|f-m|^{q} \mathbf{1}_{\{N \leq L\}}\right) \\
& =\frac{1}{Z} \int_{\{N \leq L\}}|f(x)-m|^{q} e^{-\alpha N^{p}(x)} d x \\
& \leq \frac{1}{Z} \int_{\{N \leq L\}}|f(x)-m|^{q} d x .
\end{aligned}
$$

Take

$$
m=\frac{1}{\left|B_{L_{1}}\right|} \int_{B_{L_{1}}} f(x) d x .
$$

Then, since all homogeneous norms on $\mathbb{G}$ are equivalent, by the Poincaré inequality in balls (see for example Theorem 5.6.1 of [23]) there exist constants $P_{0}, L_{1}$ such that

$$
v_{p}\left(|f-m|^{q} \mathbf{1}_{\left\{\|\cdot\|^{2} N^{p-2} \leq R\right\}} \mathbf{1}_{\{N \leq L\}}\right) \leq P_{0} e^{\alpha L_{1}^{p}} v_{p}\left|\nabla_{\mathbb{G}} f\right|^{q}
$$


Third term of (4.22): $\quad$ Set $\bar{f}=f-m$ and $A_{L, R}:=\left\{x \in \mathbb{G}:\|x\|^{2} \leq R, N(x) \geq\right.$ $L$ \}. Note that since $L>1$ we have

$$
\left\{x \in \mathbb{G}:\|x\|^{2} N^{p-2}(x) \leq R, N(x) \geq L\right\} \subset A_{L, R} .
$$

Thus

$$
v_{p}\left(|f-m|^{q} \mathbf{1}_{\left\{\|\cdot\|^{2} N^{p-2} \leq R\right\}} \mathbf{1}_{\{N \geq L\}}\right) \leq \int_{A_{L, R}}|\bar{f}(x)|^{q} d v_{p}(x) .
$$

Recall that we can write $x=(w, z) \in \mathbb{G}$ for $w \in \mathbb{R}^{n}$ and $z \in \mathbb{R}^{m}$. For $e \in\{0,1\}^{m}$, set

$$
\oint_{e}:=\left\{x=(w, z) \in \mathbb{G}:(-1)^{e_{1}} z_{1} \geq 0, \ldots,(-1)^{e_{m}} z_{m} \geq 0\right\},
$$

so that $\mathbb{G}=\cup_{e \in\{0,1\} m} S_{e}$. The reason for introducing these sets is so that in a particular $S_{e}$, the signs of $z_{j} \in \mathbb{R}$ for $j \in\{1, \ldots, m\}$ are known. By above, we then have

$$
v_{p}\left(|f-m|^{q} \mathbf{1}_{\left\{\|\cdot\|^{2} N^{p-2} \leq R\right\}} \mathbf{1}_{\{N \geq L\}}\right) \leq \sum_{e \in\{0,1\}^{m}} \int_{S_{e} \cap A_{L, R}}|\bar{f}(x)|^{q} d v_{p}(x) .
$$

We consider $\int_{S_{e} \cap A_{L, R}}|\bar{f}(x)|^{q} d v_{p}(x)$ with $e=(0, \ldots, 0)$ (the other cases are similar). Let $h \in \mathbb{G}$ be such that $\|h\|=2 \sqrt{R}$. Then we may write

$$
\begin{gathered}
\int_{\mathcal{S}_{e} \cap A_{L, R}}|\bar{f}(x)|^{q} d v_{p}(x) \\
\leq 2^{q-1} \int_{\mathcal{S}_{e} \cap A_{L, R}}|\bar{f}(x)-\bar{f}(x h)|^{q} d v_{p}(x) \\
\quad+2^{q-1} \int_{\mathcal{S}_{e} \cap A_{L, R}}|\bar{f}(x h)|^{q} d v_{p}(x) .
\end{gathered}
$$

Let $\gamma:[0, t] \rightarrow \mathbb{G}$ be a horizontal geodesic from 0 to $h$ such that $|\dot{\gamma}(s)| \leq 1$ for $s \in[0, t]$. Then, by Hölder's inequality,

$$
\begin{aligned}
& \int_{\mathcal{S}_{e} \cap A_{L, R}}|\bar{f}(x)-\bar{f}(x h)|^{q} d v_{p}(x) \\
& =\int_{S_{e} \cap A_{L, R}}\left|\int_{0}^{t} \frac{d}{d s} \bar{f}(x \gamma(s)) d s\right|^{q} d v_{p}(x) \\
& \leq t^{\frac{q}{p}} \int_{0}^{t} \int_{S_{e} \cap A_{L, R}}\left|\nabla_{\mathbb{G}} f(x \gamma(s))\right|^{q} d v_{p}(x) d s .
\end{aligned}
$$


Using this estimate in (4.26), we arrive at

$$
\begin{aligned}
& \int_{S_{e} \cap A_{L, R}}|\bar{f}(x)|^{q} d v_{p}(x) \\
& \leq 2^{q-1} d^{\frac{q}{p}}(h) \int_{0}^{t} \int_{S_{e} \cap A_{L, R}}\left|\nabla_{\mathbb{G}} f(x \gamma(s))\right|^{q} d v_{p}(x) d s \\
& \quad+2^{q-1} \int_{S_{e} \cap A_{L, R}}|\bar{f}(x h)|^{q} d v_{p}(x) .
\end{aligned}
$$

Since we have chosen $h$ such that $\|h\|=2 \sqrt{R}$, we have for $x \in A_{L, R}$

$$
\|x h\| \geq\|h\|-\|x\| \geq 2 \sqrt{R}-\sqrt{R}=\sqrt{R} .
$$

We now claim that, for fixed $R$, we can choose $h$ depending only on $R$, with $\|h\|=$ $2 \sqrt{R}$, such that for large enough $L$

$$
N^{p}(x h) \leq N^{p}(x), \quad \forall x \in S_{e} \cap A_{L, R}
$$

i.e. translation by $h$ shifts points of $S_{e} \cap A_{L, R}$ closer to the origin (with respect to the distance $N$ ).

Proof of claim (4.30): $\quad$ For $x=(w, z) \in S_{e} \cap A_{L, R}$, we have

$$
\|x\|=|w| \leq \sqrt{R}, \quad N(x) \geq L, \quad \text { and } \quad z_{1} \geq 0, \ldots, z_{m} \geq 0 .
$$

Let $h=\left(2 \sqrt{R}, 0, \ldots, 0, h_{1}, \ldots, h_{m}\right) \in \mathbb{G}=\mathbb{R}^{n+m}$, for $h_{1}, \ldots, h_{m}$ only depending on $R$ to be chosen later. Then, by the definition of the group law (see Theorem 2.2),

$$
\begin{gathered}
x h=\left(w_{1}+2 \sqrt{R}, \ldots, w_{n}, z_{1}+h_{1}+\sqrt{R}\left(\sum_{j=1}^{n} U_{1 j}^{(1)} w_{j}\right), \ldots,\right. \\
\left.z_{m}+h_{m}+\sqrt{R}\left(\sum_{j=1}^{n} U_{1 j}^{(m)} w_{j}\right)\right),
\end{gathered}
$$

so that

$$
\begin{aligned}
N^{4}(x h)-N^{4}(x)=(( & \left.\left.w_{1}+2 \sqrt{R}\right)^{2}+w_{2}^{2}+\cdots+w_{n}^{2}\right)^{2} \\
& +16\left(z_{1}+h_{1}+\sqrt{R}\left(\sum_{j=1}^{n} U_{1 j}^{(1)} w_{j}\right)\right)^{2} \\
& +\cdots+16\left(z_{m}+h_{m}+\sqrt{R}\left(\sum_{j=1}^{n} U_{1 j}^{(m)} w_{j}\right)\right)^{2} \\
& -\left(w_{1}^{2}+\cdots+w_{n}^{2}\right)^{2}-16\left(z_{1}^{2}+\cdots+z_{m}^{2}\right) .
\end{aligned}
$$


After expansion and cancellation, since we are taking $x$ such that $\|x\|=|w| \leq \sqrt{R}$, we can bound all the remaining terms in the above expression that only involve $w_{1}, \ldots, w_{n}$ from above in terms of $R$. This will leave us with

$$
\begin{aligned}
N^{4}(x h)-N^{4}(x) \leq & K(R)+32 z_{1}\left(h_{1}+\sqrt{R}\left(\sum_{j=1}^{n} U_{1 j}^{(1)} w_{j}\right)\right) \\
& +\cdots+32 z_{m}\left(h_{m}+\sqrt{R}\left(\sum_{j=1}^{n} U_{1 j}^{(m)} w_{j}\right)\right)
\end{aligned}
$$

for some constant $K$ depending on $R$ and the matrices $U^{(i)}$ for $i \in\{1, \ldots, m\}$. Now, for $i \in\{1, \ldots, m\}$ let $K_{i}(R)$ be the constant such that

$$
\sqrt{R}\left|\sum_{j=1}^{n} U_{1 j}^{(i)} w_{j}\right| \leq K_{i}(R)
$$

for all $w \in \mathbb{R}^{n}$ such that $|w| \leq \sqrt{R}$ (so that $K_{i}$ also depends on the matrix $U^{(i)}$ ). Then, since $z_{i} \geq 0$ for $i \in\{1, \ldots, m\}$ by assumption, we have

$$
N^{4}(x h)-N^{4}(x) \leq K(R)+32 z_{1}\left(h_{1}+K_{1}(R)\right)+\cdots+32 z_{m}\left(h_{m}+K_{m}(R)\right) .
$$

Let $\varepsilon>0$, and take $h_{i}=-K_{i}(R)-\varepsilon$ for $i \in\{1, \ldots, m\}$. Then

$$
N^{4}(x h)-N^{4}(x) \leq K(R)-32 \varepsilon z_{1}-\cdots-32 \varepsilon z_{m} .
$$

Now, since we are assuming that $N(x) \geq L$ and $|w| \leq \sqrt{R}$, it follows that

$$
|z|^{2} \geq \frac{1}{16}\left(L^{4}-R^{2}\right) .
$$

Thus $z_{j} \geq \frac{1}{4 \sqrt{m}}\left(L^{4}-R^{2}\right)^{\frac{1}{2}}$ for at least one $j \in\{1, \ldots, m\}$, so that by (4.31) we have

$$
N^{4}(x h)-N^{4}(x) \leq K(R)-8 \varepsilon \frac{1}{\sqrt{m}}\left(L^{4}-R^{2}\right)^{\frac{1}{2}} .
$$

For big enough $L$ the right-hand side of (4.32) is negative, which proves claim (4.30).

We now use (4.29) and (4.30) to estimate the terms of (4.28). Indeed, using (4.29) we have that

$$
\begin{aligned}
& 2^{q-1} \int_{S_{e} \cap A_{L, R}}|\bar{f}(x h)|^{q} d v_{p}(x) \\
& \quad \leq \frac{2^{q-1}}{R} \int_{S_{e} \cap A_{L, R}}|\bar{f}(x h)|^{q}\|x h\|^{2} d v_{p}(x) \\
& \quad \leq \frac{2^{q-1}}{R(L-N(h))^{p-2}} \int_{S_{e} \cap A_{L, R}}|\bar{f}(x h)|^{q}\|x h\|^{2} N^{p-2}(x h) d v_{p}(x)
\end{aligned}
$$


for $L$ large in comparison with $N(h)$. By (4.30) we also have

$$
d v_{p}(x)=Z^{-1} e^{-\alpha N^{p}(x)} d x \leq Z^{-1} e^{-\alpha N^{p}(x h)} d x=d v_{p}(x h)
$$

on $\delta_{e} \cap A_{L, R}$, so that we can continue (4.33) to see that

$$
\begin{aligned}
& 2^{q-1} \int_{S_{e} \cap A_{L, R}}|\bar{f}(x h)|^{q} d v_{p}(x) \\
& \quad \leq \frac{2^{q-1}}{R(L-N(h))^{p-2}} \int_{S_{e} \cap A_{L, R}}|\bar{f}(x h)|^{q}\|x h\|^{2} N^{p-2}(x h) d v_{p}(x h) \\
& \quad \leq \frac{2^{q-1} A}{R(L-N(h))^{p-2}} v_{p}\left|\nabla_{\mathbb{G}} f\right|^{q}+\frac{2^{q-1} B}{R(L-N(h))^{p-2}} v_{p}|f-m|^{q}
\end{aligned}
$$

where, again, we have used the translational invariance of the Lebesgue measure and Lemma 4.20.

For the first term of (4.28), note that there exists a constant $\widetilde{K}=\widetilde{K}(h)$ depending only on $h$ (and hence only on $R$ ) such that

$$
N^{p}(x \gamma(s))-N^{p}(x) \leq \widetilde{K}(h), \quad \forall x \in S_{e} \cap A_{L, R}, s \in[0, t] .
$$

This is because $N^{p}(x \gamma(s))-N^{p}(x) \rightarrow 0$ as $N(x) \rightarrow \infty$ by the mean value theorem. Then

$$
\begin{aligned}
& \int_{0}^{t} \int_{S_{e} \cap A_{L, R}}\left|\nabla_{\mathbb{G}} f(x \gamma(s))\right|^{q} d v_{p}(x) d s \\
& \quad \leq e^{\widetilde{K}(h)} \int_{0}^{t} \int_{S_{e} \cap A_{L, R}}\left|\nabla_{\mathbb{G}} f(x \gamma(s))\right|^{q} d v_{p}(x \gamma(s)) d s \\
& \quad \leq d(h) e^{\widetilde{K}(h)} v_{p}\left|\nabla_{\mathbb{G}} f\right|^{q} .
\end{aligned}
$$

Using (4.35) together with (4.34) in (4.28) yields

$$
\begin{aligned}
\int_{\mathcal{S}_{e} \cap A_{L, R}}|\bar{f}(x)|^{q} d v_{p}(x) \leq & 2^{q-1}\left(d^{\frac{q}{p}+1}(h) e^{\widetilde{K}(h)}+\frac{A}{R(L-N(h))^{p-2}}\right) v_{p}\left|\nabla_{\mathbb{G}} f\right|^{q} \\
& +\frac{2^{q-1} B}{R(L-N(h))^{p-2}} v_{p}|f-m|^{q} .
\end{aligned}
$$

The key point is that the coefficient $\frac{2^{q-1} B}{R(L-N(h))^{p-2}}$ can be made as small as we wish by taking $R$ large enough, provided $L$ remains large in comparison. Although we have done the calculations for a specific $e \in\{0,1\}^{m}$, the same may be done for arbitrary $e$ (with a different choice of $h$ ). Thus, by (4.25), we see that there exist constants $C(R, L)$ and $\delta(R, L)$ such that

$$
\begin{aligned}
& v_{p}\left(|f-m|{ }^{q} \mathbf{1}_{\left\{\|\cdot\|^{2} N^{p-2} \leq R\right\}} \mathbf{1}_{\{N \geq L\}}\right) \\
& \quad \leq C(R, L) v_{p}\left|\nabla_{\mathbb{G}} f\right|^{q}+\delta(L, R) v_{p}|f-m|^{q},
\end{aligned}
$$


where $\delta(L, R)$ may be made as small as we wish by taking $L$ and $R$ large enough. This completes the estimate of the third term of (4.22).

It remains to insert the estimates (4.23), (4.24) and (4.37) into (4.22). Doing this we arrive at

$$
\begin{aligned}
v_{p}|f-m|^{q} \leq & \left(\frac{A}{R}+P_{0}\left(L_{1}\right) e^{\alpha L_{2}^{p}}+C(R, L)\right) v_{p}\left|\nabla_{\mathbb{G}} f\right|^{q} \\
& +\left(\frac{B}{R}+\delta(R, L)\right) v_{p}|f-m|^{q},
\end{aligned}
$$

where $R$ and $L$ may be taken large enough so that $\frac{B}{R}+\delta(R, L)<1$. Upon rearrangement, this inequality, combined with the observation (4.21), proves Theorem 4.19.

Conjecture 4.21. Although our current techniques do not allow us to conclude that $\mathcal{T}_{p}$ given by (4.10) has empty essential spectrum, we conjecture that this will be true for $p>2$. This is a clear direction for further investigation.

\section{References}

[1] D. Bakry, F. Baudoin, M. Bonnefont, and D. Chafaï, On gradient bounds for the heat kernel on the Heisenberg group. J. Funct. Anal. 255 (2008), 1905-1938. MR 2462581 Zbl 1156.58009

[2] D. Bakry and M. Émery, Hypercontractivité de semi-groupes de diffusion. C. R. Acad. Sci., Paris, Sér. I Math. 299 (1984), 775-778. MR 77209 Zbl 0563.60068

[3] F. Baudoin, M. Hairer, and J. Teichmann, Ornstein-Uhlenbeck processes on Lie groups. J. Funct. Anal. 255 (2008), 877-890. MR 2433956 Zbl 1151.58018

[4] A. Bellaïche, The tangent space in sub-Riemannian geometry, In A. Bellaïche and J. J. Risler (eds.), Sub-Riemannian Geometry. Proceedings of the satellite meeting of the 1st European congress of mathematics "Journées nonholonomes: géométrie sousriemannienne, théorie du contrôle, robotique”, Paris, France, June 30-July 1, 1992, Birkhäuser, Basel, 1996, 1-78. MR 1421822 Zbl 0862.53031

[5] S. G. Bobkov and B. Zegarliński, Entropy bounds and isoperimetry. Mem. Amer. Math. Soc. 829 (2005). MR 2146071 Zbl 1161.46300

[6] A. Bonfiglioli, E. Lanconelli, and F. Uguzzoni, Stratified Lie groups and potential theory for their sub-Laplacians. Springer Monographs in Mathematics, Springer, New York, 2007. MR 2363343 Zbl 1128.43001

[7] F. Cipriani, Sobolev-Orlicz imbeddings, weak compactness, and spectrum. J. Funct. Anal. 177 (2000), 89-106. MR 1789944 Zbl 0974.46036

[8] B. Driver and T. Melcher, Hypoelliptic heat kernel inequalities on the Heisenberg group. J. Funct. Anal. 221 (2005), 340-365. MR 2124868 Zbl 1071.22005 
[9] N. Eldredge, Precise estimates for the subelliptic heat kernel on $H$-type groups. J. Math. Pures Appl. (9) 92 (2009), 52-85. MR 2541147 Zbl 1178.35096

[10] N. Eldredge, Gradient estimates for the subelliptic heat kernel on $H$-type groups. J. Funct. Anal. 258 (2010), 504-533. MR 2557945 Zbl 1185.43004

[11] G. B. Folland, Subelliptic estimates and function spaces on nilpotent Lie groups. Ark. Mat. 13 (1975), 161-207. MR 0494315 Zbl 0312.35026

[12] G. Grillo, On Persson's theorem in local Dirichlet spaces. Z. Anal. Anwendungen 17 (1998), 329-338. MR 1632559 Zbl 0908.31004

[13] A. M. Hansson and A. Laptev, Sharp spectral inequalities for the Heisenberg Laplacian. In K. Trent (ed.), Groups and analysis. The legacy of Hermann Weyl. Based on the conference in honour of Hermann Weyl, Bielefeld, Germany, September 2006. Cambridge University Press, Cambridge (U.K.), 2008, 100-115. MR 2528463 Zbl 1157.58008

[14] W. Hebisch and B. Zegarliński, Coercive inequalities on metric measure spaces. J. Funct. Anal. 258 (2010), 814-851. MR 2558178 Zbl 1189.26032

[15] A. Kaplan, Fundamental solutions for a class of hypoelliptic PDE generated by composition of quadratic forms. Trans. Amer. Math. Soc. 258 (1980), 147-153. MR 554324 Zbl 0393.35015 www.ams.org/journals/tran/1980-258-01/S0002-9947-1980-0554324-X/home.html

[16] L. D. Landau and E. M. Lifshitz, Quantum mechanics. Non-relativistic theory, Pergamon Press, New York etc., 1958. MR 0400931 Zbl 0081.22207

[17] H. Q. Li, Estimation optimale du gradient du semi-groupe de la chaleur sur le groupe de Heisenberg. J. Funct. Anal. 236 (2006), 369-394. MR 2240167 Zbl 1106.22009

[18] T. Melcher, Hypoelliptic heat kernel inequalities on Lie groups. $\mathrm{PhD}$ thesis, University of California, San Diego, 2004. MR 2705910

[19] R. Monti, Some properties of Carnot-Carathéodory balls in the Heisenberg group. Atti Accad. Naz. Lincei, Cl. Sci. Fis. Mat. Nat., IX Ser., Rend. Lincei, Mat. Appl. 11 (2000), 155-167. MR 1841689 Zbl 1197.53064

[20] A. Persson, Bounds for the discrete part of the spectrum of a semibounded Schrödinger operator. Math. Scand. 8 (1960), 143-153. MR 0133586 Zbl 0145.14901

[21] M. Reed and B. Simon, Methods of modern mathematical physics IV. Analysis of Operators. Academic Press, New York etc., 1978. MR 0493421 Zbl 0401.47001

[22] L. C. G. Rogers and D. Williams, Diffusions, Markov Processes, and Martingales 1. Foundations. Second ed. Cambridge University Press, Cambridge, 2000. MR 1796539 Zbl 0949.60003

[23] L. Saloff-Coste, Aspects of Sobolev-Type Inequalities. Cambridge University Press, Cambridge, 2002. MR 1872526 Zbl 0991.35002

[24] N. Th. Varopoulos, L. Saloff-Coste, and T. Coulhon, Analysis and Geometry on Groups. Cambridge University Press, Cambridge, 1992. MR 1218884 Zbl 1179.22009

[25] F. Y. Wang, Functional inequalities for empty essential spectrum. J. Funct. Anal. 170 (2000), 219-245. MR 1736202 Zbl 0946.58010

[26] F. Y. Wang, Functional inequalities and spectrum estimates: the infinite measure case. J. Funct. Anal. 194 (2002), 288-310. MR 1934605 Zbl 1021.58007 
[27] F. Y. Wang, Super and weak Poincaré inequalities for hypoelliptic operators. Acta Math. Appl. Sin. Engl. Ser. 25 (2009), 617-630. MR 2544981 Zbl 1185.58019 www.springerlink.com/content/5470142951515128/

[28] P.-A. Zitt, Super Poincaré inequalities: Orlicz norms and essential spectrum. Potential Anal. 35 (2011), 51-66. MR 2804552 Zbl 05935052 www.springerlink.com/content/e762065066852676/

Received May 1, 2011; revised December 7, 2011

James Inglis, INRIA Sophia-Antipolis, 2004 route des Lucioles - BP 93, 06902 Sophia Antipolis Cedex, France

E-mail: james.inglis@inria.fr 